\title{
CARACTERÍSTICAS DE LOS ENSAYOS CLÎNICOS ONCOLÓGICOS PRESENTADOS AL INSTITUTO NACIONAL DE SALUD DEL PERÚ, 1995-2019
}

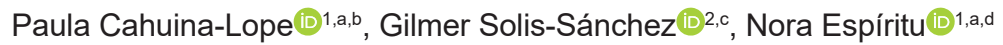 \\ ${ }^{1}$ Instituto Nacional de Salud, Lima, Perú. \\ ${ }^{2}$ Departamento de Estadística, Demografía, Humanidades y Ciencias Sociales, Facultad de Ciencias y Filosofía, Universidad \\ Peruana Cayetano Heredia, Lima, Perú. \\ a Médica cirujana; ${ }^{\mathrm{b}}$ especialista en Medicina Familiar y Comunitaria; ${ }^{\mathrm{c}}$ cirujano dentista; ${ }^{\mathrm{d}}$ especialista en Pediatría, magíster \\ de Salud Pública.
}

\section{RESUMEN}

A fin de identificar las características de los ensayos clínicos oncológicos presentados ante el Instituto Nacional de Salud del Perú en el periodo 1995-2019, se realizó un estudio observacional descriptivo, evaluando la información contenida en el Registro Peruano de Ensayos Clínicos. Se identificaron 1996 ensayos clínicos de los cuales 470 eran oncológicos (23,5\%); el 74,9\% de los ensayos clínicos oncológicos fue patrocinado principalmente por la industria farmacéutica, el $61,9 \%$ fue de fase III, siendo autorizados el $86,2 \%$. Entre los autorizados, el 55,6\% eran productos de investigación de tipo químico, el 35,9\% correspondieron a indicación terapéutica para cáncer de mama; el principal diseño fue de grupos paralelos $(84,7 \%)$, aleatorizados $(85,2 \%)$, cegados $(51,0 \%)$, el criterio de valoración principal más empleado fue la tasa de respuesta objetiva (46,7\%). Se concluye que los ensayos clínicos oncológicos se han ido incrementando con los años, con frecuencia diversa entre sus características.

Palabras clave: Características del Estudio; Cáncer; Ensayo Clínico (Fuente: DeCS BIREME).

\section{CHARACTERISTICS OF ONCOLOGICAL CLINICAL TRIALS SUBMITTED TO THE INSTITUTO NACIONAL DE SALUD DEL PERU, 1995-2019}

\begin{abstract}
A descriptive observational study was carried out to identify the characteristics of the oncological clinical trials submitted to the Instituto Nacional de Salud del Perú during the period from 1995 to 2019. The information was obtained from the Peruvian Registry of Clinical Trials. We identified 1,996 clinical trials during the studied period, from which 470 were oncological (23.5\%); $74.9 \%$ of the oncological clinical trials were mainly sponsored by the pharmaceutical industry, $61.9 \%$ were phase III and $86.2 \%$ were authorized. Regarding those authorized clinical trials, $55.6 \%$ were on chemical research products and $35.9 \%$ were on therapeutic indication for breast cancer. The most frequent study designs found were: parallel arm (84.7\%), randomized (85.2\%) and blinded (51.0\%); the most frequently used main endpoint was the objective response rate $(46.7 \%)$. We conclude that the number of oncological clinical trials has been increasing over the years, often with different characteristics.
\end{abstract}

Keywords: Study Characteristics; Neoplasms; Clinical Trial (Source: MeSH NLM).

Citar como: Cahuina-Lope P, SolisSánchez G, Espíritu N. Características de los ensayos clínicos oncológicos presentados al Instituto Nacional de Salud del Perú, 1995-2019. Rev Peru Med Exp Salud Publica. 2020;37(4):73945. doi: https://doi.org/10.17843/ rpmesp.2020.374.5167

Correspondencia: Paula Lorena Cahuina Lope; Jr. Jirón Cápac Yupanqui 1400, Jesús María, Perú; pcahuina@ins.gob.pe

Recibido: $27 / 01 / 2020$

Aprobado: 05/08/2020

En línea: 09/11/2020

\section{INTRODUCCIÓN}

Los ensayos clínicos (EC) que abordan problemas oncológicos han encontrado un escenario favorable para evaluar alternativas de manejo terapéutico y/o paliativo efectivo, tomando en cuenta los diferentes estadios de la enfermedad ${ }^{(1)}$; ello debido a que, en el contexto actual, el cáncer es un problema de salud pública de interés mundial ${ }^{(2)}$.

Algunas agencias reguladoras de EC, más allá de sus conocidas funciones de proporcionar el marco legal y resguardar la integridad de los sujetos de investigación, han implementado bases de datos abiertas donde consolidan la información de todos los EC que registran y autorizan. En este sentido, la Organización Mundial de la Salud (OMS) cuenta con el portal International Clinical Trials Registry Platform (ICTRP) (https://apps.who.int/trialsearch/Defaultaspx), a fin de asegurar el libre acceso a la información ${ }^{(3)}$. 
Esta y otras bases de datos que consolidan la información de los EC en el mundo han sido objeto de investigaciones que buscan identificar cómo ha variado la frecuencia de los EC presentados en el mundo. Viergever et al. identificaron un incremento constante en la cantidad de EC registrados, pasando de 3294 en 2004 a 23384 en el 2013, aunque estas cifras incluyen todo tipo de EC (oncológicos y no oncológicos) ${ }^{(4)}$.

En el Perú, el Instituto Nacional de Salud (INS), como autoridad reguladora de ensayos clínicos en el país, cuenta desde el 2007 con el Registro Peruano de Ensayos Clínicos (REPEC). Esta base de datos ha sido empleada por estudios previos para caracterizar los EC de forma general. Según lo reportado de forma general por Minaya et al. para el periodo 1995-2012 ${ }^{(5)}$ y por Alarcón Ruiz et al. para 1995-2017 ${ }^{(6)}$, se sabe que los EC sobre cáncer son los más frecuentes en el país.

Pese a que estas bases de datos son de dominio público para todos los investigadores, no se ha podido identificar información publicada que permita conocer aspectos relevantes de los EC orientados a patologías oncológicas en el Perú. Así, esta investigación se llevó a cabo con el objetivo de identificar las características de los ensayos clínicos oncológicos (ECO) presentados en un periodo de 25 años (1995 al 2019).

\section{EL ESTUDIO}

Se realizó un estudio observacional y descriptivo que evalúa las características de los ensayos clínicos en oncología presentados ante la Oficina General de Investigación y Transferencia Tecnológica (OGITT) del INS para su evaluación y autorización entre 1995 y el 31 de diciembre de 2019. Para la ejecución de la investigación se revisó la información pública encontrada en el REPEC, al cual se accedió libremente mediante el portal https://ensayosclinicos-repec.ins.gob.pe; la última revisión se ejecutó el 3 de enero de 2020. Los registros encontrados se consolidaron en una base de datos a fin de ser analizada.

Las variables evaluadas en los ECO se agruparon en características generales, del diseño, y del producto de investigación. Las características generales abarcaron tipo de patrocinador, estado del ensayo, número de comités de ética en investigación (CIEI) que aprobaron el ECO y número de centros de investigación donde se ejecutó el estudio.

Las alternativas del tipo de patrocinador fueron agrupadas en cinco categorías: grupos cooperativos (redes de investigación, sociedades científicas, asociaciones civiles, fundaciones y organismos de investigación), industria farmacéutica (industrias, laboratorios y compañías), institutos nacionales de salud (tanto del Perú como del extranjero) y, por último, universidades (públicas y privadas, nacionales y extranjeras). El estado del ensayo se agrupó en tres categorías: autorizado (activo, suspendido parcial, terminación anticipada, finalizado, suspendido después de la autorización,

\section{MENSAJES CLAVE}

Motivación para realizar el estudio: En el Perú, se presentan ensayos clínicos para la evaluación de productos farmacéuticos y dispositivos médicos en diferentes especialidades, con un incremento en la presentación de ensayos oncológicos, ello motivado por el incremento en la prevalencia global del cáncer. Es necesario conocer las características que estos han tenido en los últimos 25 años.

Principales hallazgos: Existe variación en la cantidad de ensayos clínicos presentados. Se ha observado un aumento en los ensayos oncológicos, la mayoría de ellos patrocinados por la industria farmacéutica y principalmente enfocados a los tipos más prevalentes de neoplasias como el cáncer de mama.

Implicancias: El conocimiento de las características de los ensayos clínicos oncológicos permitirá a los profesionales de salud identificar la dirección que está tomando el desarrollo terapéutico en medicina.

cancelado), no autorizado, otros (trámite sin efecto, suspendidos antes de obtener autorización, declarados en abandono, declarados como desistimiento, declarado improcedente, en evaluación).

Sobre las características del diseño de los ECO, las variables consideradas fueron la fase del ensayo, el diseño específico, el tipo de cegamiento, el tipo de aleatorización y el criterio de valoración principal utilizado. En lo referente a las características del producto de investigación se consideró el tipo de producto y su indicación.

Tanto las características del diseño como las del producto de investigación se analizaron para el total de ECO presentados y para los ensayos autorizados. Los datos no encontrados se consideraron «no registrados» (NR).

Los datos recolectados se evaluaron con el programa estadístico Stata V16.1 (Stata Corporation, College Station, Texas, EUA) y con medidas de resumen descriptivo de proporciones (\%) para las variables cualitativas. No se realizaron procesos de imputación estadística ni pruebas inferenciales.

La realización del estudio no requirió la aprobación de un comité de ética, por tratarse de un análisis de base secundaria, de dominio público, de libre acceso y por no contener datos que permitan identificar a los sujetos de investigación.

\section{HALLAZGOS}

Se identificaron 1996 EC durante el periodo 1995-2019, de los cuales, 470 fueron oncológicos (23,5\%); los primeros 
registros se presentaron en 1999 ( $\mathrm{n}=8 ; 1,7 \%)$. Existe variabilidad en la cantidad de ECO presentados por año; se evidenció que entre 1999 y 2002 hubo menos de 20 por año, tras lo cual se presentó un incremento constante hasta 2008, año en el que se presentó el pico máximo $(n=50 ; 10,6 \%)$ y se redujeron progresivamente los años siguientes. A pesar de ello, la proporción de ECO presentados respecto al total ha mostrado un ascenso en el periodo evaluado (Figura 1).

En cuanto a las características generales de los ECO, se encontró que el tipo de patrocinador más frecuente correspondía a la industria farmacéutica (74,9\%), la mayoría autorizados ( $\mathrm{n}=405 ; 86,2 \%)$, de los cuales el $61,2 \%$ se encontraba finalizado $(n=248)$. Cerca de la mitad $(49,6 \%)$ de los ECO fueron aprobados por solo un CIEI, siendo el más frecuente ( $\mathrm{n}=249 ; 26,7 \%$ ) el del Instituto Nacional de Enfermedades Neoplásicas (INEN). El 38,1\% de los ECO presentados se ejecutó en un único centro de investigación, hecho que fue el más frecuente, aunque se identificó que esta cifra podía llegar hasta 14 centros de investigación (Tabla 1). La proporción de los ECO autorizados ha variado con los años, con una tendencia de irregular descenso (Material suplementario).

Sobre las características del diseño, la mayoría de los ECO eran de fase III, seguido por los de fase II; el diseño específico más usado es el de grupos paralelos (84,5\%), los estudios menos frecuentes fueron los adaptativos (basket $=0,4 \%$, umbre$l l a=0,2)$. Entre los ECO autorizados, se encontró similitud entre los que presentaban tipo de cegamiento de etiqueta abierta y los de doble ciego (47,9 y 46,4\%; respectivamente), además, se tuvo que el 85,2\% presentaba aleatorización (Tabla 2).

En el análisis de las características del producto de investigación, se encontró que los de tipo químico fueron los más frecuentes entre los autorizados $(55,6 \%)$. Sobre la indicación se encontró que la mayoría era terapéutica $(91,4 \%)$, se destacan las orientadas al cáncer de mama $(35,9 \%)$; entre las indicaciones paliativas, las de emesis $(45,7 \%)$ y dolor $(34,3 \%)$ fueron las más frecuentes. El criterio de valoración principal más empleado fue la tasa de respuesta objetiva, alcanzó el $47,0 \%$ de todos los ensayos presentados y el $46,7 \%$ entre todos los autorizados (Tabla 3).
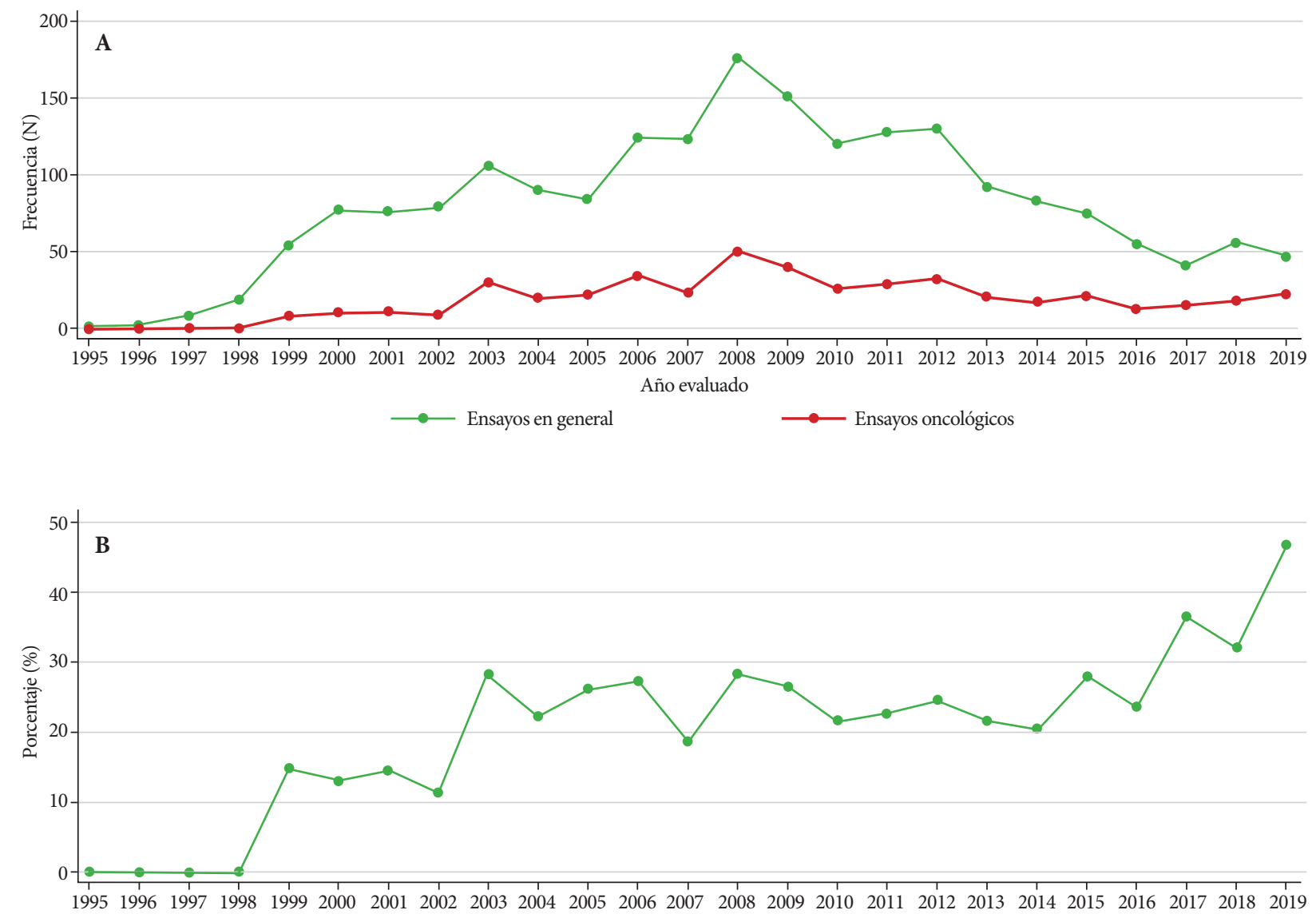

Año evaluado

Figura 1. A. Frecuencia de ensayos clínicos en general y oncológicos presentados según año evaluado. B. Proporción de ensayos clínicos oncológicos según año durante el periodo 1995-2019. 
Tabla 1. Características generales de los ensayos clínicos oncológicos presentados durante el periodo 1995-2019.

\begin{tabular}{|c|c|}
\hline Características & n (\%) \\
\hline \multicolumn{2}{|l|}{ Tipo de patrocinador } \\
\hline Grupos cooperativos & $54(11,5)$ \\
\hline Industria farmacéutica & $352(74,9)$ \\
\hline Institutos nacionales de salud & $5(1,1)$ \\
\hline Universidades & $2(0,4)$ \\
\hline Otros & $3(0,6)$ \\
\hline NR & $54(11,5)$ \\
\hline \multicolumn{2}{|l|}{ Estado del Ensayo } \\
\hline Autorizado & $405(86,2)$ \\
\hline Activo & $88(18,7)$ \\
\hline Suspendido parcial & $1(0,2)$ \\
\hline Terminación anticipada & $13(2,8)$ \\
\hline Finalizado & $248(52,8)$ \\
\hline Suspendido después de la autorización & $48(10,2)$ \\
\hline Cancelado & $7(1,5)$ \\
\hline No autorizado & $18(3,8)$ \\
\hline Otros & $47(10,0)$ \\
\hline Trámite sin efecto & $13(2,8)$ \\
\hline $\begin{array}{l}\text { Suspendidos antes de obtener } \\
\text { autorización }\end{array}$ & $4(0,9)$ \\
\hline Declarados en abandono & $10(2,1)$ \\
\hline Declarados como desistimiento & $13(2,8)$ \\
\hline Declarado improcedente & $1(0,2)$ \\
\hline En evaluación & $6(1,3)$ \\
\hline \multicolumn{2}{|l|}{ Número de CIEI que aprobaron el protocolo } \\
\hline 1 & $233(49,6)$ \\
\hline 2 & $0(0,0)$ \\
\hline 3 & $101(21,5)$ \\
\hline 4 & $78(16,6)$ \\
\hline 5 & $37(7,9)$ \\
\hline 6 & $15(3,2)$ \\
\hline 7 & $3(0,6)$ \\
\hline 8 & $2(0,4)$ \\
\hline 9 & $1(0,2)$ \\
\hline \multicolumn{2}{|l|}{ Número de centros de investigación } \\
\hline 1 & $179(38,1)$ \\
\hline 2 & $81(17,2)$ \\
\hline 3 & $70(14,9)$ \\
\hline 4 & $49(10,4)$ \\
\hline 5 & $36(7,7)$ \\
\hline 6 & $18(3,8)$ \\
\hline 7 & $14(3,0)$ \\
\hline 8 & $10(2,1)$ \\
\hline 9 & $5(1,1)$ \\
\hline 10 & $3(0,6)$ \\
\hline 11 & $2(0,4)$ \\
\hline 12 & $0(0,0)$ \\
\hline 13 & $1(0,2)$ \\
\hline 14 & $2(0,4)$ \\
\hline Total & $470(100,0)$ \\
\hline
\end{tabular}

NR: No registrado

CIEI: Comité Institucional de Ética en Investigación.
Tabla 2. Características del diseño de los ensayos clínicos oncológicos presentados y autorizados durante el periodo 1995-2019.

\begin{tabular}{lrr}
\hline Características & $\begin{array}{c}\text { Presentados } \\
\mathbf{n}(\%)\end{array}$ & $\begin{array}{c}\text { Autorizados } \\
\mathbf{n}(\%)\end{array}$ \\
\hline Fase del ensayo & & \\
I & $18(3,8)$ & $14(3,5)$ \\
I-II & $1(0,2)$ & $1(0,2)$ \\
II & $135(28,7)$ & $119(29,4)$ \\
II-III & $1(0,2)$ & $1(0,2)$ \\
III & $289(61,5)$ & $246(60,7)$ \\
IV & $22(4,7)$ & $20(4,9)$ \\
NR & $4(0,9)$ & $4(1,0)$
\end{tabular}

Diseño específico

De un solo brazo

$59(12,6)$

$51(12,6)$

Grupos paralelos

$397(84,5)$

$343(84,7)$

Cruzado

$7(1,5)$

$6(1,5)$

Factorial

Adaptativo-Basket

$4(0,9)$

$2(0,5)$

Adaptativo-Umbrella

$2(0,5)$

$2(0,4)$

$1(0,2)$

Tipo de cegamiento

Etiqueta abierta
Simple
Doble
Triple

NR

$1(0,2)$

$\begin{array}{rr}222(47,2) & 194(47,9) \\ 13(2,8) & 10(2,5)\end{array}$

$222(47,2) \quad 188(46,4)$

$5(1,1) \quad 5(1,2)$

$8(1,7) \quad 8(2,0)$

Tipo de aleatorización

No aleatorizado

$9(1,9) \quad 7(1,7)$

Aleatorizado

$400(85,1) \quad 345(85,2)$

No aplica

$61(13,0)$

$53(13,1)$

Criterio de valoración principal

$\begin{array}{lrr}\text { Calidad de vida } & 1(0,2) & 1(0,2) \\ \text { Incidencia de cáncer } & 2(0,4) & 2(0,5) \\ \begin{array}{l}\text { Reporte de síntomas } \\ \text { Seguridad }\end{array} & 10(2,1) & 10(2,5) \\ \begin{array}{l}\text { Sensibilidad y } \\ \text { especificidad }\end{array} & 23(4,9) & 22(5,4) \\ \begin{array}{l}\text { Sobrevida global } \\ \begin{array}{l}\text { Sobrevida libre de } \\ \text { enfermedad }\end{array}\end{array} & 2(0,4) & 2(0,5) \\ \begin{array}{l}\text { Sobrevida libre de eventos } \\ \begin{array}{l}\text { Sobrevida libre de } \\ \text { progresión }\end{array}\end{array} & 5(13,4) & 54(13,3) \\ \begin{array}{l}\text { Tasa de respuesta completa } \\ \text { Tasa de respuesta objetiva }\end{array} & 8(1,3) & 18(4,4) \\ \begin{array}{l}\text { Tiempo hasta la progresión } \\ \text { otal }\end{array} & 9(1,7) & 5(1,2) \\ & 470(100) & 89(22,0) \\ \end{array}$

NR: No registrado 
Tabla 3. Características del producto de investigación de los ensayos clínicos oncológicos presentados y autorizados durante el periodo 1995-2019.

\begin{tabular}{|c|c|c|}
\hline Características & $\begin{array}{c}\text { Presentados } \\
\text { n (\%) }\end{array}$ & $\begin{array}{c}\text { Autorizados } \\
\text { n (\%) }\end{array}$ \\
\hline \multicolumn{3}{|l|}{ Tipo de producto } \\
\hline Dispositivo médico & $3(0,6)$ & $2(0,5)$ \\
\hline Producto químico & $263(56,0)$ & $225(55,6)$ \\
\hline Producto biológico & $196(41,7)$ & $172(42,5)$ \\
\hline Otros & $8(1,7)$ & $6(1,5)$ \\
\hline \multicolumn{3}{|l|}{ Indicación del producto } \\
\hline Paliativo & $38(8,1)$ & $35(8,6)$ \\
\hline Anemia & $3(7,9)$ & $2(5,7)$ \\
\hline Disfunción hepática & $1(2,6)$ & $1(2,9)$ \\
\hline Disfunción intestinal & $2(5,3)$ & $2(5,7)$ \\
\hline Dolor & $12(31,6)$ & $12(34,3)$ \\
\hline Emesis & $18(47,4)$ & $16(45,7)$ \\
\hline Hipercalcemia & $1(2,6)$ & $1(2,9)$ \\
\hline Neumonía & $1(2,6)$ & $1(2,9)$ \\
\hline Terapéutico & $432(91,9)$ & $370(91,4)$ \\
\hline Anal & $1(0,2)$ & $0(0,0)$ \\
\hline Cabeza y cuello & $10(2,3)$ & $7(1,9)$ \\
\hline Cerebro & $1(0,2)$ & $1(0,3)$ \\
\hline Colorrectal & $12(2,8)$ & $9(2,4)$ \\
\hline Cuello uterino & $7(1,6)$ & $4(1,1)$ \\
\hline Esofágico & $3(0,7)$ & $3(0,8)$ \\
\hline Gastrointestinal & $24(5,6)$ & $22(5,9)$ \\
\hline Hematológico & $32(7,4)$ & $29(7,8)$ \\
\hline Hígado & $7(1,6)$ & $6(1,6)$ \\
\hline Linfático & $36(8,3)$ & $28(7,6)$ \\
\hline Mama & $148(34,3)$ & $133(35,9)$ \\
\hline Múltiple & $13(3,0)$ & $10(2,7)$ \\
\hline Nasofaríngeo & $1(0,2)$ & $1(0,3)$ \\
\hline Osteosarcoma & $1(0,2)$ & $1(0,3)$ \\
\hline Ovario & $10(2,3)$ & $7(1,9)$ \\
\hline Páncreas & $8(1,9)$ & $6(1,6)$ \\
\hline Piel & $10(2,3)$ & $8(2,2)$ \\
\hline Próstata & $28(6,5)$ & $22(5,9)$ \\
\hline Pulmonar & $66(15,3)$ & $59(15,9)$ \\
\hline Renal & $4(0,9)$ & $4(1,1)$ \\
\hline Sarcoma mieloide & $2(0,5)$ & $2(0,5)$ \\
\hline Tiroides & $2(0,5)$ & $2(0,5)$ \\
\hline Útero & $1(0,2)$ & $1(0,3)$ \\
\hline Vagina y vulva & $1(0,2)$ & $1(0,3)$ \\
\hline Vejiga & $4(0,9)$ & $4(1,1)$ \\
\hline Total & $470(100)$ & $405(100)$ \\
\hline
\end{tabular}

\section{DISCUSIÓN}

Se identificó que la cantidad de ECO presentados ha variado anualmente. No obstante, considerando la proporción de estos respecto a la totalidad de EC registrados, se evidenció una tendencia ascendente, donde para el 2019 estos representaban casi el 50\%. Este comportamiento no solo es propio en el Perú, ya que según lo registrado en ClinicalTrials.gov y EudraCT, podemos encontrar distribuciones similares en otros países ${ }^{(7)}$.

En el periodo de 25 años, en el cual se realizó la evaluación, se encontró que el $23,5 \%$ de los EC registrados eran oncológicos; cifra similar al 22,4\% reportado por Minaya et al. ${ }^{(5)}$ para el periodo 1995-2012. Estos resultados dan cuenta de la importante presencia que mantiene este tipo de investigaciones en nuestro país a lo largo del tiempo, levemente mayor al 21,8\% encontrado en ClinicalTrials.gov para el periodo $2007-2010^{(8)}$.

Por otro lado, los productos de investigación estudiados fueron mayoritariamente de indicación terapéutica (91,9\%), orientados a atender neoplasias de mama y pulmón. Estos hallazgos concuerdan con el análisis de Minaya et al. ${ }^{(5)}$, y responden a la alta incidencia de estos tipos de cáncer en el mundo ${ }^{(9)}$. La alta morbimortalidad del cáncer ha promovido también ECO de índole diagnóstico, siendo un claro ejemplo el estudio de la eficacia de solución de tinción mediada por el receptor de folato como herramienta de detección temprana de cáncer de cuello uterino ${ }^{(10)}$, o el uso de nuevos equipos de «tomosíntesis digital» para la identificación sistemática de cáncer de mama ${ }^{(11)}$.

En cuanto al patrocinio de los EC presentados en el Perú, en su mayoría por la industria farmacéutica; similar patrón se evidencia en los países del oeste europeo, donde para el periodo 2007-2015 se encontró que el 74\% de los EC presentaban patrocinio comercial ${ }^{(12)}$. La participación de la industria farmacéutica en la investigación de nuevas drogas oncológicas no es un hecho novedoso, ya se tiene identificado que este tipo de financiamiento ha mostrado resultados favorables en estos estudios ${ }^{(13)}$.

Durante los dos últimos años se registraron dos nuevos diseños para ECO; estos diseños adaptativos o llamados Masters Protocols, correspondieron al Basket (definido por cohortes con diferentes tipos de tumores asignados a un mismo tratamiento), y Umbrella (definido por cohortes con presencia de un mismo tipo tumoral con o sin biomarcadores asociados que reciben diferentes tratamientos) ${ }^{(14,15)}$. Estos diseños adaptativos, juntamente con el de tipo Platform (aún no registrado en nuestro país), se vienen adoptando en el mundo de forma creciente desde el 2001 como alternativa para los ECO; siendo estos más frecuente en Estados Unidos ${ }^{(16)}$.

De forma clásica, los criterios de valoración principal empleados en los ECO correspondieron a medidas de beneficio clínico; donde se considera la mejora de signos y/o sín- 
tomas, y de la calidad de vida del paciente ${ }^{(17)}$. Sin embargo, durante la última década los patrocinadores han utilizado otros criterios para alcanzar la aprobación acelerada de medicamentos contra el cáncer, entre estas tenemos a la evaluación de la reducción tumoral y el retraso en el crecimiento tumoral ${ }^{(18)}$; ante esta situación las agencias reguladoras se han encargado de valorar la pertinencia, caso a caso, del uso de criterios de valoración sustitutos y de brindar directrices que guíen su uso razonable ${ }^{(19)}$. Todo ello, ha orientado que actualmente los criterios de valoración principal empleados para estos estudios sean la tasa de respuesta objetiva, o sobrevida libre de progresión, considerando que se debe de tomar en cuenta la pertinencia de su utilización, y que siempre dependerá del contexto de la enfermedad y la magnitud del efecto, entre otros factores ${ }^{(20)}$.

Los resultados que se reportan en este estudio deben de considerar que la información proviene de lo registrado en el REPEC, cuya plataforma se creó en 2007, y que para entonces recopiló de forma retrospectiva los datos de los EC que anteriormente habían sido evaluados y autorizados por el Ministerio de Salud. Ello conlleva a que exista un latente sesgo de información para el periodo 1995-2006. Pese a esta limitación, el presente trabajo caracteriza al grupo de EC más frecuente que se han presentado en nuestro país en los últimos 25 años.

En conclusión, la frecuencia de los ECO que se han presentado en el Perú muestra variaciones en el periodo evalua- do, aunque proporcionalmente se ha evidenciado un comportamiento ascendente con los años. Se logró identificar también que los ECO de fase II y III son los más frecuentes en nuestro país. En cuanto al tipo de indicación del producto de investigación, se evidenció que una gran proporción era terapéutico y orientado a cáncer de mama.

La identificación de ECO con diseños adaptativos representa un desafío para los comités de ética, pacientes, investigadores y autoridades regulatorias en general. Por ese motivo, en cumplimiento de su papel de ente rector en materia de EC, el INS es el llamado a establecer actividades de capacitación que garanticen su adecuada implementación en nuestro país.

Contribuciones de autoría: Todos los autores participaron en la concepción y diseño del artículo, y en la recolección de datos. GS realizó el análisis estadístico de datos. Todos los autores participaron en la interpretación de los datos, redactaron el artículo, hicieron la revisión crítica del manuscrito y aprobaron su versión final.

Financiamiento: Autofinanciado.

Conflictos de interés: Paula Cahuina-Lope es coordinadora del equipo de evaluación de ensayos clínicos de la Oficina Ejecutiva de Investigación, Nora Espíritu (al momento del envío del artículo) era Directora General de la Oficina General de Investigación y Transferencia Tecnológica. Gilmer Solis es consultor externo del Instituto Nacional de Salud.

Material suplementario: Disponible en la versión electrónica de la RPMESP.

\section{REFERENCIAS BIBLIOGRÁFICAS}

1. Sotelo-Rodríguez DC, Ruíz-Patiño A, Ricaurte L, Arrieta O, Zatarain-Barón ZL, Cardona AF. Challenges and shifting paradigms in clinical trials in oncology: the case for immunological and targeted therapies. Ecancer. 2019;13:936. doi: 10.3332/ecancer.2019.936.

2. Unger JM, CookE, Tai E, Bleyer A. The Role of Clinical Trial Participation in Cancer Research: Barriers, Evidence, and Strategies. Am Soc Clin Oncol Educ Book. 2016;36:185-98. doi: 10.1200/EDBK_156686.

3. Ogino $\mathrm{D}$, Takahashi $\mathrm{K}$, Sato $\mathrm{H}$. Characteristics of clinical trial websites: information distribution between ClinicalTrials.gov and 13 primary registries in the WHO registry network. Trials. 2014;15(1):428. doi: 10.1186/1745-6215-15-428.

4. Viergever RF, Li K. Trends in global clinical trial registration: an analysis of numbers of registered clinical trials in different parts of the world from 2004 to 2013. BMJ Open. 2015;5(9):e008932. doi: 10.1136/bmjopen-2015-008932.

5. Minaya G, Fuentes D, Obregón C, Ayala-Quintanilla B, Yagui M. Características de los ensayos clínicos autorizados en el Perú, 1995-2012. Rev Peru Med Exp Salud Publica. 2012;29(4):431-36. doi: 10.1590/s172646342012000400003.

6. Alarcon-Ruiz CA, Roque-Roque JS, Heredia P, Góme-Briceño AR, Quispe AM. Twenty-two years' experience registering trials in a low-middle income country: The Peruvian Clinical Trial Registry. J Evid Based Med. 2019;12(3):187-93. doi: 10.1111/jebm.12354.

7. The Lancet Oncology. Clinical trial registry reporting: a transparent solution needed. The Lancet Oncology. 2019;20(6):741. doi: 10.1016/ S1470-2045(19)30350-X.
8. Hirsch BR, Califf RM, Cheng SK, Tasneem A, Horton J, Chiswell K, et al Characteristics of Oncology Clinical Trials: Insights From a Systematic Analysis of ClinicalTrials.gov. JAMA Intern Med. 2013;173(11):972. doi: 10.1001/jamainternmed.2013.627.

9. Bray F, Ferlay J, Soerjomataram I, Siegel RL, Torre LA, Jemal A. Global cancer statistics 2018: GLOBOCAN estimates of incidence and mortality worldwide for 36 cancers in 185 countries. CA Cancer J Clin. 2018;68(6):394-424. doi: 10.3322/caac.21492.

10. Zhao Y, Li M, Li Y, Lv Q, Chen F, Li B, et al. Evaluation of Folate Receptor-Mediated Cervical Dyeing as a Method for Detection of Cervical Lesions: Journal of Lower Genital Tract Disease. 2019;23(2):133-7. doi: 10.1097/LGT.0000000000000411.

11. SOS Study team, Chauvie S, De Maggi A, Baralis I, Dalmasso F, Berchialla P, et al. Artificial intelligence and radiomics enhance the positive predictive value of digital chest tomosynthesis for lung cancer detection within SOS clinical trial. Eur Radiol. 2020;30(7):4134-40. doi: 10.1007/s00330-020-06783-z.

12. Dombernowsky T, Hædersdal M, Lassen U, Thomsen SF. Development in the number of clinical trial applications in Western Europe from 2007 to 2015: retrospective study of data from national competent authorities. BMJ Open. 2017;7(7):e015579. doi: 10.1136/bmjopen-2016-015579.

13. Al-Badriyeh D, Alameri M, Al-Okka R. Cost-effectiveness research in cancer therapy: a systematic review of literature trends, methods and the influence of funding. BMJ Open. 2017;7(1):e012648. doi: 10.1136/ bmjopen-2016-012648.

14. Goldberg RM, Wei L, Fernandez S. The Evolution of Clinical Trials in Oncology: Defining Who Benefits from New Drugs Using Innovative 
Study Designs. The Oncol. 2017;22(9):1015-9. doi: 10.1634/theoncologist.2017-0153.

15. Sudhop T, Brun NC, Riedel C, Rosso A, Broich K, Senderovitz T. Master protocols in clinical trials: a universal Swiss Army knife? The Lancet Oncology. 2019;20(6):e336-42. doi: 10.1016/S14702045(19)30271-2.

16. Park JJH, Siden E, Zoratti MJ, Dron L, Harari O, Singer J, et al. Systematic review of basket trials, umbrella trials, and platform trials: a landscape analysis of master protocols. Trials. 2019;20(1):572. doi: 10.1186/s13063019-3664-1.

17. Kilickap S, Demirci U, Karadurmus N, Dogan M, Akinci B, Sendur MAN. Endpoints in oncology clinical trials. JBUON. 2018; 23(s1):1-6.
18. Wilson MK, Karakasis K, Oza AM. Outcomes and endpoints in trials of cancer treatment: the past, present, and future. The Lancet Oncology. 2015;16(1):e32-42. doi: 10.1016/S1470-2045(14)70375-4.

19. Food and Drug Administration. Clinical Trial Endpoints for the Approval of Cancer Drugs and Biologics [Internet]. Maryland: Estados Unidos. Departamento de Salud y Servicios Sociales; 2018. Disponible en: https:// www.fda.gov/media/71195/download.

20. Kamat AM, Sylvester RJ, Böhle A, Palou J, Lamm DL, Brausi M, et al. Definitions, End Points, and Clinical Trial Designs for Non-Muscle-Invasive Bladder Cancer: Recommendations From the International Bladder Cancer Group. JCO. 2016;34(16):1935-44. doi: 10.1200/JCO.2015.64.4070. 